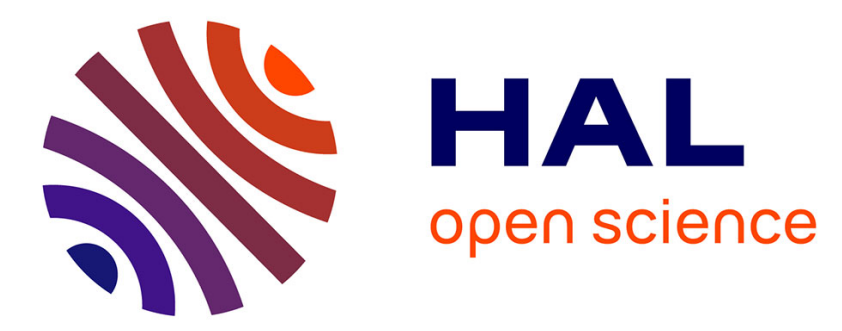

\title{
Temperature and time dependence of the field-driven magnetization steps in Ca3Co2O6 single crystals
}

V. Hardy, M.R. Lees, O.A. Petrenko, D.Mck. Paul, Delphine Flahaut, S. Hebert, A. Maignan

\section{- To cite this version:}

V. Hardy, M.R. Lees, O.A. Petrenko, D.Mck. Paul, Delphine Flahaut, et al.. Temperature and time dependence of the field-driven magnetization steps in Ca3Co2O6 single crystals. Physical Review B: Condensed Matter and Materials Physics (1998-2015), 2004, 70 (6), pp.064424-1-064424-7. 10.1103/PhysRevB.70.064424 . hal-01499383

\section{HAL Id: hal-01499383 https://hal.science/hal-01499383}

Submitted on 19 Jan 2022

HAL is a multi-disciplinary open access archive for the deposit and dissemination of scientific research documents, whether they are published or not. The documents may come from teaching and research institutions in France or abroad, or from public or private research centers.
L'archive ouverte pluridisciplinaire HAL, est destinée au dépôt et à la diffusion de documents scientifiques de niveau recherche, publiés ou non, émanant des établissements d'enseignement et de recherche français ou étrangers, des laboratoires publics ou privés. 


\title{
Temperature and time dependence of the field-driven magnetization steps in $\mathrm{Ca}_{3} \mathrm{Co}_{2} \mathrm{O}_{6}$ single crystals
}

\author{
V. Hardy,$^{1,2}$ M. R. Lees, ${ }^{1}$ O. A. Petrenko, ${ }^{1}$ D. McK. Paul, ${ }^{1}$ D. Flahaut,${ }^{2}$ S. Hébert,${ }^{2}$ and A. Maignan ${ }^{2}$ \\ ${ }^{1}$ Department of Physics, University of Warwick, Coventry CV4 7AL, United Kingdom \\ ${ }^{2}$ Laboratoire CRISMAT, UMR 6508, Boulevard du Maréchal Juin, 14050, Caen Cedex, France \\ (Received 7 January 2004; revised manuscript received 15 April 2004; published 31 August 2004)
}

\begin{abstract}
For the spin-chain compound $\mathrm{Ca}_{3} \mathrm{Co}_{2} \mathrm{O}_{6}$, the magnetization curves as a function of the magnetic field are strongly out-of-equilibrium at low temperature, and they exhibit several steps whose origins are still a matter for debate. In the present paper we report on a detailed investigation of the temperature and time dependence of these features. First, it is found that some of the magnetization steps can disappear as the characteristic time of the measurement is increased. A comparison of the influence of temperature and time points to the existence of a thermally activated process that plays an important role in determining the form of the magnetization curves. Second, direct investigations of the magnetic response as a function of time show that this thermally activated process competes with a second relaxation mechanism of a very different nature, which becomes dominant at the lowest temperatures. These results shed new light on the peculiar magnetization process of this geometrically frustrated, Ising-like spin-chain compound.
\end{abstract}

DOI: 10.1103/PhysRevB.70.064424

PACS number(s): 75.60. $-\mathrm{d}, 75.45+\mathrm{j}$

\section{INTRODUCTION}

Magnetic compounds combining low dimensionality and geometrical frustration can give rise to complex physical properties. One of the best-known examples is provided by the $\mathrm{CsCoX}_{3}$ compounds, where $\mathrm{X}$ is $\mathrm{Cl}$ or $\mathrm{Br}^{1}{ }^{1}$ In these Ising one-dimensional (1D) materials, the spin-chains are arranged on a triangular lattice, while both the intrachain and interchain couplings are antiferromagnetic. This situation induces geometrical frustration and unusual magnetic behavior. For instance, the magnetization curves of $\mathrm{CsCoCl}_{3}$ at low temperatures show transitions around $33 \mathrm{~T}$ and $44 \mathrm{~T}$, that have been ascribed to a complex combination of intrachain and interchain spin rearrangements. ${ }^{2}$

Recently, another family of 1D compounds in which the chains form a triangular lattice has attracted considerable attention. Its general formula is $\mathrm{A}_{3}{ }_{3} \mathrm{ABO}_{6}$, where $\mathrm{A}^{\prime}$ is $\mathrm{Ca}$ or $\mathrm{Sr}$, while A and B are transition metal elements. ${ }^{3}$ Some members of this family possess a pronounced Ising-like character with the spins parallel to the chains, while the intrachain and interchain coupling is ferromagnetic and antiferromagnetic, respectively. ${ }^{4-7} \mathrm{Ca}_{3} \mathrm{Co}_{2} \mathrm{O}_{6}$ is the one of these geometrically frustrated compounds that has been the most intensively studied in recent years. ${ }^{4,8-15}$

$\mathrm{Ca}_{3} \mathrm{Co}_{2} \mathrm{O}_{6}$ has a rhombohedral structure composed of $\left[\mathrm{Co}_{2} \mathrm{O}_{6}\right]_{\infty}$ infinite chains running along the $c$ axis of the corresponding hexagonal cell, with the $\mathrm{Ca}$ cations located in between them. ${ }^{16}$ The chains are made of alternating, facesharing $\mathrm{CoO}_{6}$ trigonal prisms and $\mathrm{CoO}_{6}$ octahedra. Each chain is surrounded by six equally spaced chains forming a triangular lattice in the $a b$ plane (see inset of Fig. 1). The intrachain Co-Co separation is $\sim 0.26 \mathrm{~nm}$, while the interchain distance is $\sim 0.52 \mathrm{~nm}^{16}$

In $\mathrm{Ca}_{3} \mathrm{Co}_{2} \mathrm{O}_{6}$, the intrachain coupling is ferromagnetic while the interchain coupling (much weaker) is antiferromagnetic. ${ }^{4}$ In the paramagnetic regime, the crystals used in the present study exhibit a Curie-Weiss temperature
$\theta_{C W} \simeq+80 \mathrm{~K}$ (in line with the previous studies with $H \|_{c}$ ), ${ }^{9,11}$ which confirms the strong ferromagnetic character of the intrachain coupling. The electronic states of the Co ions at the two sites (trigonal prisms and octahedra) are not yet perfectly established, but to date, the most widely accepted scheme is the following: (i) both Co ions are $\mathrm{Co}^{3+}\left(3 d^{6}\right)$; (ii) the splitting of the $3 d$ electronic levels induced by the crystalline electric field (CEF) are very different at the two Co sites. The upper doublet is separated by a gap that is expected to be significantly larger for the octahedral environment than for the prismatic one. ${ }^{4}$ This must favor the high-spin state $(S$ $=2$ ) for the trigonal prisms and the low-spin state $(S=0)$ for the octahedra. As discussed by Aasland et al., ${ }^{4}$ this picture appears to be the most likely when considering all the physi-

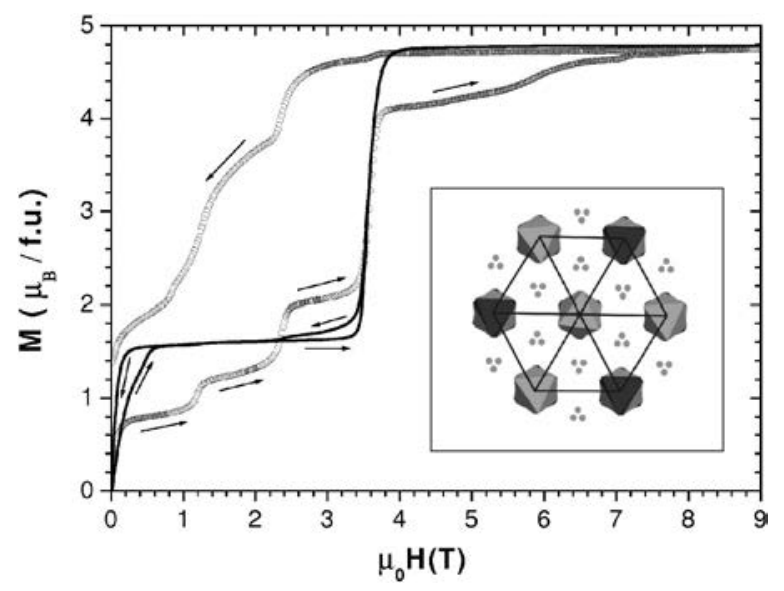

FIG. 1. Hysteresis loops recorded with a sweep rate of $0.1 \mathrm{~T} / \mathrm{min}$ at $2 \mathrm{~K}$ (circles) and $10 \mathrm{~K}$ (solid line). The arrows indicate the direction of the field variation. The inset shows a projection of the structure along the hexagonal $c$-axis (the dark and light polyhedra represent $\mathrm{CoO}_{6}$ trigonal prisms and $\mathrm{CoO}_{6}$ octahedra, respectively; the grey circles represent the $\mathrm{Ca}$ ions; the solid lines emphasize the triangular arrangement of the chains in the $a b$ plane). 
cal properties (effective moment $\mu_{e f f}$, saturation magnetization $M_{\text {sat }}$, neutron diffraction data). There are, however, other combinations of electronic states that cannot be ruled out at this time. ${ }^{4,16}$ The magnetic measurements carried out with $H \| c$ on the crystals used in the present study show $\left(\mu_{\text {eff }} / \mu_{B}\right)^{2} \simeq 37$ and $M_{\text {sat }} / \mu_{B} \simeq 4.8$ per formula unit. This set of values is consistent with an alternation between $S=2$ (at the prismatic sites) and $S=0$ (at the octahedral sites), if one considers for the former site a Landé factor $g \simeq 2.4$ [the corresponding theoretical values are $\left(\mu_{\text {eff }} / \mu_{B}\right)^{2} \simeq 35$ and $M_{\text {sat }} / \mu_{B} \simeq 4.8$ per formula unit]. Note that such a large $g$ value is not unreasonable, since the orbital contribution is often found to be only partially quenched for $\mathrm{Co}^{3+}\left(3 d^{6}\right){ }^{4,9}$ On the other hand, alternative pictures involving a fraction of intermediate-spin $(S=1)$ or high-spin $(S=2)$ states at the octahedral sites can also account for the magnetic data. ${ }^{11}$ It is clear that more sophisticated investigations will have to be carried out to resolve this issue (e.g., x-ray magnetic dichroism experiments on our crystals are in progress).

The complete spin Hamiltonian relevant to $\mathrm{Ca}_{3} \mathrm{Co}_{2} \mathrm{O}_{6}$ has yet to be established. The presence of a large difference in the magnetic susceptibility for the field applied parallel and perpendicular to $c$, which has been observed at all temperatures up to $300 \mathrm{~K},{ }^{9}$ suggests that the single-ion anisotropy related to the $\mathrm{CEF}$ at the prismatic sites must be a leading term in this spin Hamiltonian. In addition, the ferromagnetic intrachain coupling, $J$, and the anti-ferromagnetic interchain coupling, $J^{\prime}$, must be taken into account, as it is this combination that is at the origin of the $3 \mathrm{D}$ magnetic order at low temperature. ${ }^{4}$ The hexagonal arrangement of the chains generates geometrical frustration, which prevents this order from being complete. Whether or not a model with interactions that have a strictly Ising character can produce an adequate description of the magnetic properties of $\mathrm{Ca}_{3} \mathrm{Co}_{2} \mathrm{O}_{6}$ remains to be seen. Alternatively, interactions between the spin components perpendicular to the $c$-axis may need to be taken into account. The degree of one-dimensionality $\left(J / J^{\prime}\right.$ ratio) has yet to be unambiguously established. In a magnetic field, a Zeeman term must also be included. This external polarization adds another degree of complexity to the interplay between the first three terms, which generates unusual behavior in magnetic fields. ${ }^{4,8-15}$

In a zero field, the main features of the magnetism in $\mathrm{Ca}_{3} \mathrm{Co}_{2} \mathrm{O}_{6}$ are the following: (i) a long-range spin ordering driven by the antiferromagnetic interchain coupling takes place at $T_{N} \simeq 26 \mathrm{~K} ;{ }^{4}$ (ii) the system only reaches a "partially disordered antiferromagnetic" state (PDA) at low $T$, because of the geometrical frustration. ${ }^{9}$ In the PDA state, ${ }^{17}$ two thirds of the ferromagnetic chains are coupled antiferromagnetically, while the remaining third remain incoherent (disordered chains with zero net magnetization).

$\mathrm{Ca}_{3} \mathrm{Co}_{2} \mathrm{O}_{6}$ shows striking $M(H)$ curves for $T<T_{N}$ : at "high" temperatures, e.g., $10 \mathrm{~K}$, there is a single, large magnetization step, which has been attributed to a ferrimagnetic to ferromagnetic transition; ${ }^{4}$ at the lowest temperatures, e.g., $2 \mathrm{~K}$, several new steps appear in the $M(H)$ curves. ${ }^{9,11}$ The origin of these latter features is still controversial at the present time. In this paper we report on an investigation of the role of temperature and time on the onset of these magnetization steps.

\section{EXPERIMENTAL DETAILS}

Single crystals of $\mathrm{Ca}_{3} \mathrm{Co}_{2} \mathrm{O}_{6}$ were grown using a flux method described previously. ${ }^{11}$ They have the shape of short hexagonal rods terminating in three diamondlike faces at each end. Because of their small size $\left(\sim 0.7 \times 0.7 \times 1 \mathrm{~mm}^{3}\right)$, we have used a set of four crystals (the same as that used in the heat capacity study reported in Ref. 14). The magnetic field was applied parallel to the $c$-axis, i.e., the direction of the chains and of the spins, by using the alignment procedure described in Ref. 14. Approximating the shape of the crystals by an ellipsoid, ${ }^{18}$ the demagnetizing factor $N$ along the $c$ direction was estimated to be $\sim 0.25$. When the magnetization is large, this shape effect can induce a sizeable difference between the applied external field $\mu_{0} H$ and the average internal field $B$, since $B=\mu_{0}[H+(1-N) M]$. The present study is focused on a regime where the magnetization is of the order of $M_{\text {sat }} / 3 \sim 1.6 \mu_{B} /$ f.u. $\sim 1.2 \times 10^{5} \mathrm{~A} / \mathrm{m}$, inducing a shift between $B$ and $\mu_{0} H$ which remains limited to $\sim 0.1 \mathrm{~T}$. The magnetization curves $M(H)$ were recorded after zerofield cooling from $T=35 \mathrm{~K}$ (i.e., a temperature larger than $T_{N}$ ), by using a Vibrating Sample Magnetometer (Oxford Instruments) equipped with a $12 \mathrm{~T}$ magnet, with different magnetic field sweep rates (from $0.01 \mathrm{~T} / \mathrm{min}$ to $1 \mathrm{~T} / \mathrm{min}$ ). We also recorded curves of magnetic relaxation $M(t)$ in fixed fields, by using a Superconducting Quantum Interference Device magnetometer (Quantum Design) in fields of up to $5 \mathrm{~T}$. This study was carried out on each branch of the $M(H)$ loops. In all cases, the crystals were first zero-field-cooled from $35 \mathrm{~K}$ down to the measuring temperature. Then, the measurement field was directly applied in the case of the field-increasing (FI) branch, whereas it was installed after the crystals had been held in a field of $5 \mathrm{~T}$ for $1 \mathrm{~min}$ in the case of the field-decreasing (FD) branch (in $5 \mathrm{~T}$, the magnetization is saturated for $T \geqslant 4 \mathrm{~K}$ ). The magnetization was then recorded as a function of time for at least $10^{4}$ seconds. The stability of the temperature over this time interval was found to be better than $0.2 \%$. Curves of $M$-vs- $T$ for the range of magnetic fields investigated show that such a temperature drift has a very limited impact on $M\left(\Delta M / M<10^{-4}\right.$ in all cases). Therefore, the observed variation of $M$ along the relaxation curves is an effect of time.

\section{RESULTS}

Figure 1 shows hysteresis loops recorded at 2 and $10 \mathrm{~K}$, with a sweep rate of $0.1 \mathrm{~T} / \mathrm{min}$. The shape of these curves is in line with the existing literature. ${ }^{9,11}$ When the field is increased at $10 \mathrm{~K}$, the magnetization rapidly reaches a first plateau, before being switched to the saturation value $\left(M_{\text {sat }}\right.$ $\sim 4.8 \mu_{B}$ /f.u.) above $H_{C} \simeq 3.6 \mathrm{~T}$. The magnetization on the plateau is equal to $\sim M_{s a t} / 3$, a feature that has been ascribed to the formation of a ferrimagnetic state, consisting of ferromagnetic chains with two thirds of them having spins up and one third having spins down. ${ }^{4}$ The magnetization jump to $M_{\text {sat }}$ at $H_{C}$ was attributed to a ferrimagnetic-to-ferromagnetic transition. ${ }^{4}$ The reverse leg of the loop at $10 \mathrm{~K}$ shows that hysteresis is present just below $H_{C}$ and when approaching a zero field. As $T$ is decreased below $10 \mathrm{~K}$, the hysteresis in- 

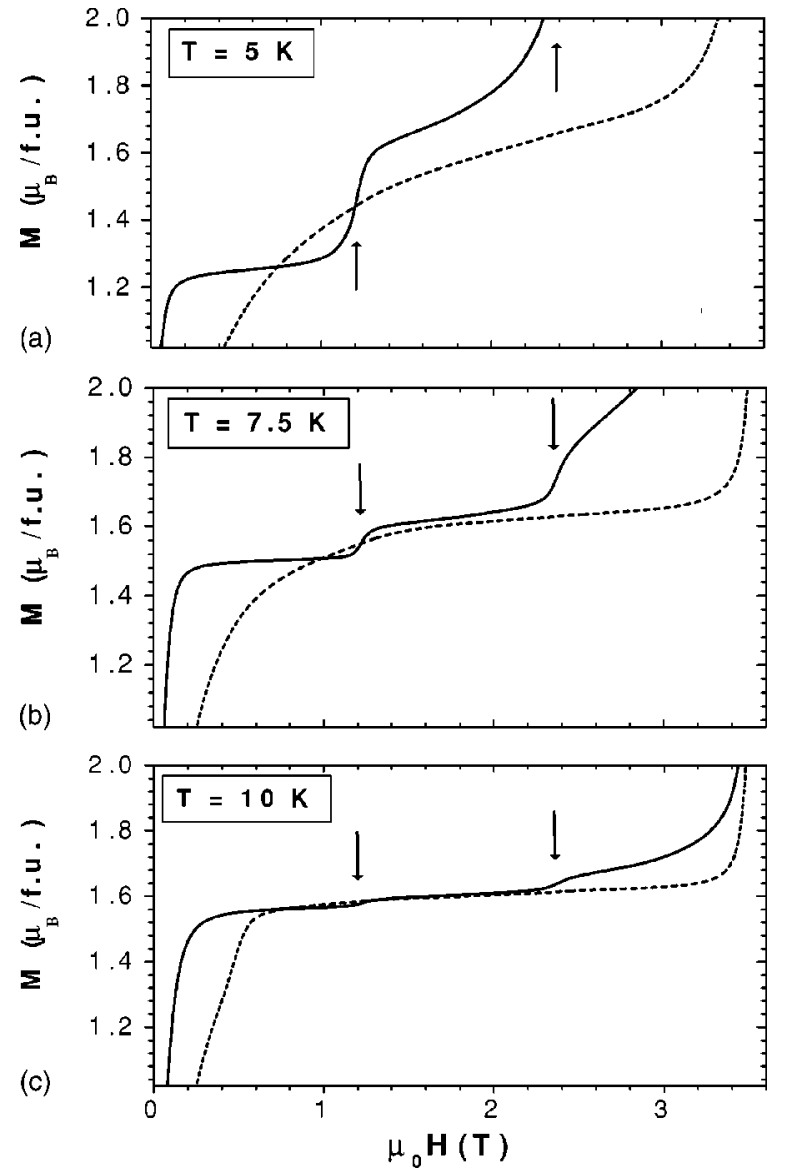

FIG. 2. Enlargements of hysteresis loops recorded with a sweep rate of $0.1 \mathrm{~T} / \mathrm{min}$ at $5 \mathrm{~K}(\mathrm{a}), 7.5 \mathrm{~K}$ (b) and $10 \mathrm{~K}$ (c). The dashed lines and solid lines are the field-increasing and field-decreasing branches, respectively. The arrows denote the step fields $H_{S 1}$ and $H_{S 2}$ (see text).

creases and occupies the whole field range, while new magnetization steps appear at low fields. The curve at $2 \mathrm{~K}$ in Fig. 1 illustrates the peculiar magnetic behavior found at very low- $T$ in $\mathrm{Ca}_{3} \mathrm{Co}_{2} \mathrm{O}_{6}$. Below $H_{C} \simeq 3.6 \mathrm{~T}$, one can clearly observe two additional magnetization steps at $H_{S 1} \sim 1.2 \mathrm{~T}$ and $H_{S 2} \sim 2.4 \mathrm{~T}$. The transition at $H_{C}$ is still present, but $M$ does not reach $M_{\text {sat }}$. As $H$ is further increased above $H_{C}$, less pronounced steps in the magnetization can be detected at characteristic fields of about $4.7 \mathrm{~T}, 5.9 \mathrm{~T}$ and $7.1 \mathrm{~T}$ by examining the derivative $d M / d H$. The saturation magnetization is reached at $8.5 \mathrm{~T}$. As the field is decreased, clear signatures of the characteristic fields $H_{C}, H_{S 1}$ and $H_{S 2}$ are observed in the magnetization curve (an additional small maximum is also found at $\sim 0.85 \mathrm{~T}$ in the derivative of the reverse leg). In this very low- $T$ regime $(T \ll 10 \mathrm{~K})$, the hysteresis persists down to the zero field, leading to a large remanent magnetization.

Let us now consider the intermediate temperature range, $2 \mathrm{~K} \leqslant T \leqslant 10 \mathrm{~K}$, in more detail. Figure 2 shows enlargements of $M(H)$ curves around the low-field plateau(s), at various temperatures between 5 and $10 \mathrm{~K}$. On the FI branch, the additional steps at $H_{S 1}$ and $H_{S 2}$ rapidly disappear as the temperature is increased (e.g., they are no longer detectable at
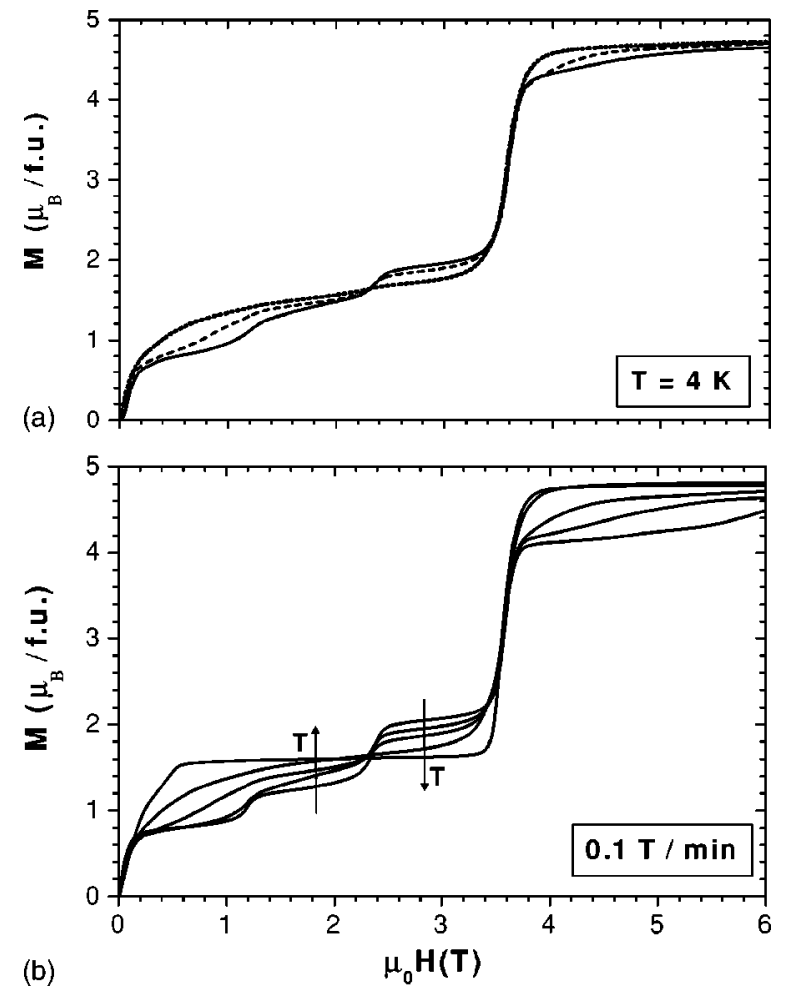

FIG. 3. (a) Field-increasing branches of loops recorded at $4 \mathrm{~K}$ with different sweep rates: $1 \mathrm{~T} / \mathrm{min}$ (solid line), $0.1 \mathrm{~T} / \mathrm{min}$ (dashed line) and $0.01 \mathrm{~T} / \mathrm{min}$ (dotted line); (b) field-increasing branches of loops recorded with a sweep rate of $0.1 \mathrm{~T} / \mathrm{min}$ at various temperatures $(2,3,4,5$ and $10 \mathrm{~K})$. The arrows indicate the ordering of these curves on each side of $H_{S 2}$.

$5 \mathrm{~K}$ ). In contrast, they remain clearly visible on the FD branch up to much higher temperatures (e.g., they are still detectable at $10 \mathrm{~K}$ ). It is worth noting that the values of the critical fields $H_{S 1} \sim 1.2 \mathrm{~T}$ and $H_{S 2} \sim 2.4 \mathrm{~T}$ are the same at all temperatures.

Figure 3(a) shows the FI branches of the $M(H)$ curves recorded with various magnetic-field sweep rates at the intermediate temperature $T=4 \mathrm{~K}$. The steps at $H_{S 1}$ and $H_{S 2}$ are clearly visible for the fastest sweep rate $(1 \mathrm{~T} / \mathrm{min})$. As the sweep rate is decreased, these features are progressively smoothed. The steps in $M(H)$ are still detectable for $0.1 \mathrm{~T} / \mathrm{min}$, but they have completely disappeared for $0.01 \mathrm{~T} / \mathrm{min}$. One observes that all three curves cross at the same point, close to $H_{S 2}$.

Figure 3(b) shows the FI branches of $M(H)$ curves recorded at different temperatures around $4 \mathrm{~K}$ (from 2 to $10 \mathrm{~K})$, with the same sweep rate $(0.1 \mathrm{~T} / \mathrm{min})$. As $T$ is increased, the steps are progressively washed out, and the magnetization curve below $H_{C}$ tends to a single plateau at $\sim M_{\text {sat }} / 3$. One also observes that the curves for all temperatures cross at the same point, which is very close to that seen in Fig. 3(a).

Figure 4 shows the hysteresis loop recorded at $4 \mathrm{~K}$ with $0.01 \mathrm{~T} / \mathrm{min}$, along with that obtained at $5 \mathrm{~K}$ with $0.1 \mathrm{~T} / \mathrm{min}$. Remarkably, the curves are very similar to one another. They exhibit the same features, including a peculiar crossing between the field-increasing and field-decreasing curves around 


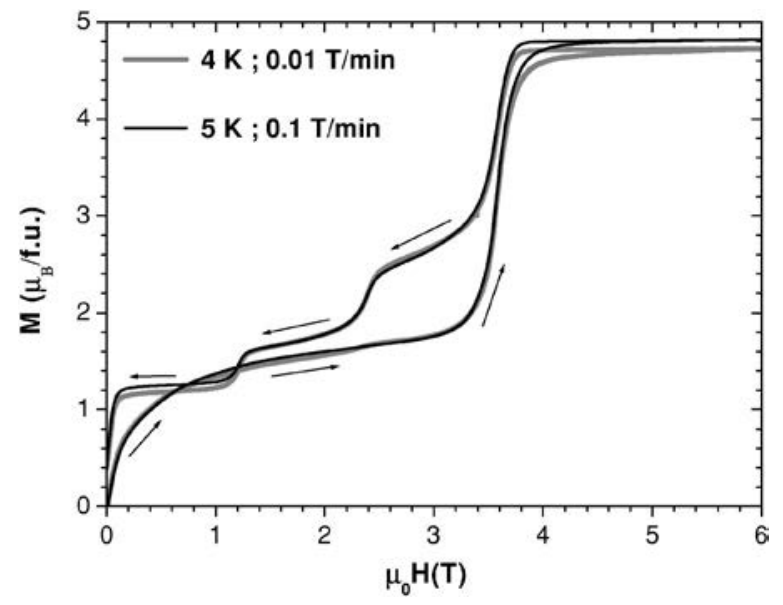

FIG. 4. Hysteresis loops recorded at $4 \mathrm{~K}$ with $0.01 \mathrm{~T} / \mathrm{min}$ (grey line) and at $5 \mathrm{~K}$ with $0.1 \mathrm{~T} / \mathrm{min}$ (black line). The arrows indicate the direction of the field variation.

1 T. The almost perfect superposition of the curves in Fig. 4 reveals a strong interplay between the effects of time and temperature.

In order to gain a deeper insight into the time effects present in this material, we have directly recorded magnetic relaxation curves, $M(t)$, at various temperatures in the range $2-10 \mathrm{~K}$ and in various magnetic fields lower than $H_{C}$. We have observed very different behavior at $2 \mathrm{~K}$ and $10 \mathrm{~K}$ : (i) at $10 \mathrm{~K}$, there is almost no relaxation in the field range where $M \sim M_{\text {sat }} / 3$. When $M$ is away from this ferrimagnetic plateau (for instance, around $3 \mathrm{~T}$ on the FD branch), the relaxation drives $M$ towards $M_{s a t} / 3$; (ii) at $2 \mathrm{~K}$, the relaxation is more pronounced and is qualitatively very different: Whatever the magnetic field, $M$ increases with time on the FI branch, while it decreases with time on the FD branch.

The most complex features are found in the relaxation curves at intermediate temperatures, in between 2 and $10 \mathrm{~K}$. This is illustrated in Fig 5(a) which shows relaxation curves recorded at $4 K$, on the FI and FD branches, in several magnetic fields around $H_{S 2}$. On the FD branch, $M$ decreases with time for all fields around $H_{S 2} \cdot{ }^{19}$ On the FI branch, the situation is more complex since the magnetization at $4 \mathrm{~K}$ increases with time for $H<H_{S 2}$ (e.g., $2 \mathrm{~T}$ ), whereas it decreases with time for $H>H_{S 2}$ (e.g., $2.7 \mathrm{~T}$ ). Such a change in the sign of the relaxation above $H_{S 2}$ is typical of the intermediate temperatures close to $4 \mathrm{~K}$. As shown in Fig. 5(a), the magnetization at $2 \mathrm{~K}$ still increases with time in a field of $2.7 \mathrm{~T}$. It should be noted that the field dependence of the $M(t)$ curves at $4 \mathrm{~K}$ is consistent with the influence of the sweep rate value on the VSM data, since the magnetization steps at $H_{S 1}$ and $H_{S 2}$ in Fig. 3(a) are found to disappear as the timescale of the experiment is increased. Furthermore, the $M(t)$ curves reveal an intricate time-dependence at $4 \mathrm{~K}$ for the fields close to $H_{S 2}$ on the FI branch. This is more visible in Fig 5(b) which shows the normalized relaxation curves $M(t) / M_{0}$, for fields around $H_{S 2}$, where $M_{0}$ is the magnetization value measured about $25 \mathrm{~s}$ after the end of the field installation. In $2.3 \mathrm{~T}$ for instance, one observes a striking nonmonotonic behavior: $M$ first increases in the short-time
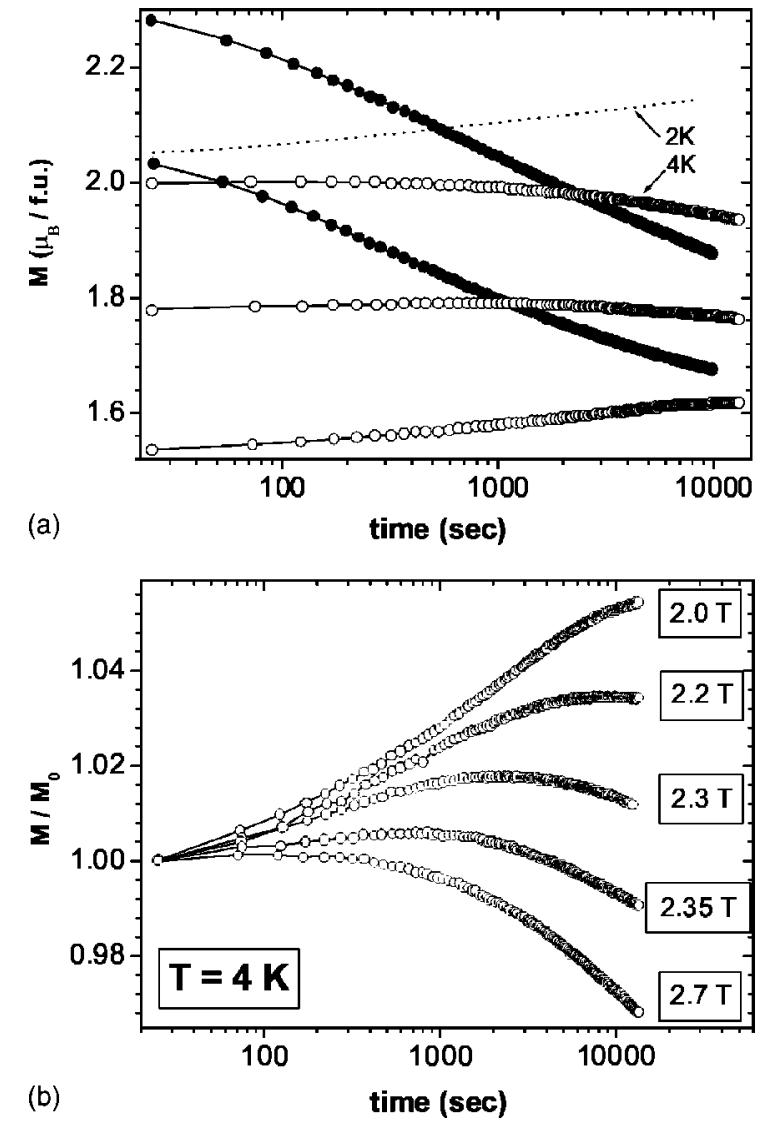

FIG. 5. Relaxation curves recorded at $4 \mathrm{~K}$ in different magnetic fields: in (a), the open circles correspond to the field-increasing branch (2.0, 2.3 and $2.7 \mathrm{~T}$ from bottom to top) and the filled circles correspond to the field-decreasing branch $(2.0$ and $2.3 \mathrm{~T}$ from bottom to top). The dotted line shows the relaxation at $2 \mathrm{~K}$ for $2.7 \mathrm{~T}$ on the field-increasing branch; (b) shows normalized relaxation curves at $4 \mathrm{~K}$ (using the first measurement taken about $25 \mathrm{~s}$. after the end of the field installation), which were recorded on the fieldincreasing branch in different magnetic fields around $H_{S 2}$.

range, before decreasing at longer-time. These crossovers take place at times as long as several thousand seconds.

\section{DISCUSSION}

The nature of the magnetic states on the plateaux separated by $H_{S 1}$ and $H_{S 2}$ is not yet clear. The most direct technique to address such an issue, i.e. neutron diffraction experiments as a function of the magnetic field, have not been performed to date, since large enough single crystals are not yet available. The present magnetization measurements, however, provide us with new qualitative information about the crossover with temperature between a regime showing only one large step at $H_{C}($ e.g., $10 \mathrm{~K})$, and a low- $T$ regime with the additional magnetization steps at $H_{S 1}$ and $H_{S 2}$ (e.g., $2 \mathrm{~K})$.

Despite a striking change in the global shape of $M(H)$ between 10 and $2 \mathrm{~K}$ (Fig. 1), a close look at the data demonstrates that the magnetic behavior at these two temperatures remain closely related to each other: Beyond the clear 
persistence of the transition at $H_{C}$ down to $2 \mathrm{~K}$, the present data reveals that the step fields at $H_{S 1}$ and $H_{S 2}$ can still be detected at $10 \mathrm{~K}$ on the reverse leg of the loop [Fig. 2(c)]. These results suggest a continuous crossover between 2 and $10 \mathrm{~K}$, rather than the occurrence of a true critical temperature within this $T$ range. Furthermore, our results demonstrate that this crossover is not only driven by the temperature but is also strongly dependent on time.

One of the main findings of the present study is indeed the metastable character of the magnetization steps that occur below $H_{C}$. The measurements at $4 \mathrm{~K}$ show that the steps at $H_{S 1}$ and $H_{S 2}$ progressively disappear with time, and that the magnetization actually tends to the ferrimagnetic plateau $\left(M_{s a t} / 3\right)$ on both branches of the loops. This behavior suggests that the ferrimagnetic state remains the true ground state for $H<H_{C}$, even at the lowest $T$. Furthermore, the similar roles played by temperature and time indicates that the relaxation mechanism pushing $M$ to $M_{\text {sat }} / 3$ is a thermally activated process. This is spectacularly confirmed by Fig. 4 where it is shown that a variation in temperature can be exactly counterbalanced by a variation in the timescale of the measurement.

The $M(t)$ relaxation curves, recorded for fixed values of $H$ and $T$, were found to be consistent with the VSM data. At high- $T$ (e.g., $10 \mathrm{~K}), M(t)$ systematically tends to $M_{\text {sat }} / 3$ (i.e., for all values of $H$ and on both the FI and FD branches of the loops). At low- $T$ (e.g., $2 \mathrm{~K}$ ), the $M(t)$ curves exhibit a very different behavior, since the magnetization is systematically found to increase with time on the FI branch, while it decreases on the FD leg. It must be emphasized that this type of relaxation acting at very low- $T$ can move $M$ away from $M_{\text {sat }} / 3$. For instance, at $2 \mathrm{~K}$ on the FI branch, the initial magnetization in $3 \mathrm{~T}$ is $\sim 2.10 \mu_{B}$ /f.u. (i.e., larger than $M_{\text {sat }} / 3 \sim 1.6 \mu_{B}$ /f.u.), and $M$ increases with time up to $\sim 2.22 \mu_{B}$ /f.u. for $t \sim 10^{4} \mathrm{~s}$.

On the basis of our results, we propose that the shape of the $M(H)$ curves of $\mathrm{Ca}_{3} \mathrm{Co}_{2} \mathrm{O}_{6}$ for $T \leqslant 10 \mathrm{~K}$ and $H \leqslant H_{C}$ results from a combination of two relaxation processes: (1) a mechanism by which $M$ increases with time on the FI branch and decreases with time on the FD branch, for all field values; (2) a standard thermally activated relaxation by which $M$ tends to the $3 \mathrm{D}$ equilibrium value (i.e., $M_{\text {sat }} / 3$ for $\left.H<H_{c}\right)$.

A thermally activated phenomenon like process (2) becomes less and less efficient in overcoming the barriers as $T$ is decreased. One can consider that only process (1) is at work for standard "experiment times" at $2 \mathrm{~K}$. At "high" temperatures (e.g., $10 \mathrm{~K})$, the opposite situation applies. Process (2) is dominant, leading to a fast setting of the equilibrium ferrimagnetic state on both branches of the $M(H)$ loops. Once it is installed, this long-range ordered, stable state, can impede the effect of process (1). The intermediate regime around $T=4 \mathrm{~K}$ corresponds to the peculiar situation where the two relaxation processes have a comparable influence over the value of $M$ within the experimental time-window.

Let us now comment on the possible origins of each of these relaxation processes. Several features observed at very low $T$ (e.g., $2 \mathrm{~K}$ ) suggest that process (1) could be related to the phenomenon of Quantum Tunneling of Magnetization
(QTM). ${ }^{20,21}$ In particular, it is found that the step fields are regularly spaced and are associated with enhancements in the value of the relaxation rate. ${ }^{22}$ Note that a roughly constant spacing between the steps $(\sim 1.2 \mathrm{~T})$ is still observed if one considers the average internal field $B$ (see Sec. II). The observation of the QTM requires rather large spin values and a strong uni-axial anisotropy. ${ }^{20}$ Such features exist in $\mathrm{Ca}_{3} \mathrm{Co}_{2} \mathrm{O}_{6}$, owing to its Ising-like nature and the presence of $\mathrm{Co}^{3+}$ ions in the high spin-state $(S=2)$. The occurrence of QTM in $\mathrm{Ca}_{3} \mathrm{Co}_{2} \mathrm{O}_{6}$ will have to be further tested by carrying out complementary experiments (e.g., by investigating the expected influence of an additional transverse magnetic field) ${ }^{20}$ As for process (2), it is very likely that this corresponds to establishing the ferrimagnetic state (two chains "up" and one chain "down") through a series of collective spin reversals along some of the chains. For a frustrated, Ising-like spin-chain compound, such a mechanism has to face sizeable energy barriers which can lead to a freezing of the spins as $T$ is decreased. ${ }^{23}$ The existence of a Frozen Spin state with $T_{F S} \sim 5-7 \mathrm{~K}$ has been reported in previous studies of $\mathrm{Ca}_{3} \mathrm{Co}_{2} \mathrm{O}_{6} \cdot{ }^{8,9,14,15}$ Below $T_{F S}$, the global freezing of the spin system hinders process (2), which allows the development of the magnetization steps via process (1).

Let us now comment on the crossing point found in the $M(H)$ plane for curves recorded with different sweep rates at $4 \mathrm{~K}$ and different temperatures around $4 \mathrm{~K}$ (Fig. 3). In spite of its striking character, this fixed point of approximate coordinates $\left(H_{s 2 ;} M_{s a t} / 3\right)$ may be seen as a "natural" consequence of the fact that the magnetization plateaus related to QTM at $H_{s 2}$ turn out to be located around the 3D equilibrium value, i.e. $M_{\text {sat }} / 3$. The point is that the values of the magnetization plateaux between each resonance field have no special significance within the framework of a QTM process (i.e. they are essentially governed by the tunneling rate and the magnetic field sweep rate). ${ }^{20}$ The crossing found for different sweep rates at $4 \mathrm{~K}$ [Fig. 3(a)] may be seen as an interplay between the two relaxation processes: The $M(H)$ curve at short time shows steps related to QTM (in particular across $M_{s a t} / 3$ at $H_{s 2}$ ), while the thermally activated relaxation process drives $M$ toward $M_{\text {sat }} / 3$ as a function of time. Accordingly, $M$ increases with time for $H<H_{s 2}$ [where $\left.M(t \sim 0)<M_{\text {sat }} / 3\right]$ whereas it decreases with time for $H>H_{s 2}$ [where $M(t \sim 0)>M_{s a t} / 3$ ]. This leads to a crossing point close to $\left(H_{s 2 ;} ; M_{s a t} / 3\right)$. Since this behavior is related to a thermally activated mechanism, a crossing at the same point is also expected for curves recorded at different temperatures around $4 \mathrm{~K}$ [Fig. 3(b)]. It should be emphasized that there is no similar crossing point at $H_{s 1}$, which can be ascribed to the fact that $M(t \sim 0)<M_{\text {sat }} / 3$ for both magnetization plateaux related to QTM around this magnetic field.

The shape of a $M(t)$ curve driven by QTM is known to be potentially affected by the evolution of the internal field $B$ during the relaxation. ${ }^{24}$ To our knowledge, however, this effect cannot account for a change in the sign of the relaxation rate. On the other hand, the picture given above, based on the interplay between two independent relaxation processes (namely, QTM towards $M_{\text {sat }}$ and a collective, thermally activated process towards $M_{s a t} / 3$ ), can give rise to complex $M(t)$ curves if this competition operates over the experimen- 
tal time window. In fields close to $H_{s 2}, M(t \sim 0)$ at $4 \mathrm{~K}$ is slightly larger than $M_{\text {sat }} / 3$. Therefore, the QTM makes $M$ increase with time whereas the thermally activated relaxation makes $M$ decrease with time. The combination of these effects can give rise to nonmonotonic $M(t)$ curves such as those reported in Fig. 5(b).

It must be noted that significant differences were found between the FI and FD branches. In particular, the magnetization steps at $H_{S 1}$ and $H_{S 2}$ remain visible at a much higher temperature on the FD branch than on the FI leg (see Fig. 2). On the FD branch, the spin system starts from a fully polarized state, for which the ferromagnetic intrachain coupling is totally satisfied, in contrast to the FI branch which starts from the PDA state. ${ }^{17}$ Accordingly, the onset of the ferrimagnetic state on the FD branch requires the complete reversal of some of the ferromagnetic chains (from the "up" to the "down" configuration), whereas it only involves a ferromagnetic alignment of incoherent chains in the case of the FI branch. The energy cost associated with the first spin rever$s a l$ is thus lower in the latter case. This may explain why the thermally activated process leading to the ferrimagnetic state is more efficient on the FI branch.

\section{CONCLUSION}

For $T$ lower than the 3D ordering temperature $T_{N} \simeq 26 \mathrm{~K}$, the frustrated, Ising-like spin-chain compound $\mathrm{Ca}_{3} \mathrm{Co}_{2} \mathrm{O}_{6}$ exhibits two different types of $M(H)$ curves when the field is applied along the $c$ axis (direction of the chains and of the spins). In the high- $T$ range (illustrated by $T=10 \mathrm{~K}$ ), $M(H)$ is principally characterized by a magnetization jump at $H_{C}$ $\simeq 3.6 \mathrm{~T}$, between $M_{\text {sat }} / 3$ and $M_{\text {sat }}\left(M_{\text {sat }}\right.$ being the saturation magnetization). In the low- $T$ range (illustrated by $T=2 \mathrm{~K}$ ), $M(H)$ becomes strongly hysteretic and exhibits several additional magnetization steps. These new features are particu- larly pronounced on the field-increasing branch, for fields lower than $H_{C}$.

Our investigation of the magnetization as a function of temperature and time suggests that there is a continuous crossover between these two regimes, which is driven not only by the temperature but also depends on time. More precisely, we propose that the various shapes of the $M(H)$ curves found for $T<T_{N}$ and $H<H_{C}$ are the result of a competition between two relaxation mechanisms.

(1) A $T$-independent relaxation process, leading to a magnetization increase on the field-increasing branch and a decrease on the field-decreasing branch. This process may correspond to spin-flips related to the phenomenon of the Quantum Tunneling of Magnetization.

(2) A thermally activated relaxation process, pushing $M$ to $M_{\text {sat }} / 3$ on both branches of the $M(H)$ cycles. This process is associated with the onset of the ferrimagnetic state, which develops by domino-like spin reversals along the chains.

At high- $T$ (e.g., $10 \mathrm{~K}$ ), the spin system is rapidly driven into the ferrimagnetic state through process (2), while, at low-T (e.g., $2 \mathrm{~K}$ ), the spin freezing (which starts developing below $T_{F S} \sim 5-7 \mathrm{~K}$ ) allows process (1) to generate magnetization steps for regularly spaced field values. At intermediate temperatures close to $T=4 \mathrm{~K}$, the influence of each of these two processes becomes comparable. The combination of their effects can account for (i) the fact that the appearance of additional steps in the $M(H)$ curves depends on the timescale of the experiments; (ii) the observation of unusual nonmonotonic relaxation curves.

Local investigations of the spin ordering and spin dynamics in $\mathrm{Ca}_{3} \mathrm{Co}_{2} \mathrm{O}_{6}$ are highly desirable to confirm this scenario.

We acknowledge the financial support of the EPSRC (United Kingdom) and the DRI of CNRS (France) for this project.
${ }^{1}$ M. F. Collins and O. A. Petrenko, Can. J. Phys. 75, 605 (1997), and references therein.

${ }^{2}$ K. Amaya, H. Hori, I. Shiozaki, M. Date, M. Ishizuka, T. Sakakibara, T. Goto, N. Miura, H. Kikuchi, and Y. Ajiro, J. Phys. Soc. Jpn. 59, 1810 (1990).

${ }^{3}$ K. E. Stitzer, J. Darriet, and H.-C. zur Loye, Curr. Opin. Solid State Mater. Sci. 5, 535 (2001).

${ }^{4}$ S. Aasland, H. Fjellvag, and B. Hauback, Solid State Commun. 101, 187 (1997).

${ }^{5}$ H. Kageyama, K. Yoshimura, and K. Kosuge, J. Solid State Chem. 140, 14 (1998).

${ }^{6}$ S. Niitaka, H. Kageyama, M. Kato, K. Yoshimura, and K. Kosuge, J. Solid State Chem. 146, 137 (1999); S. Niitaka et al., J. Phys. Soc. Jpn. 70, 1222 (2001).

${ }^{7}$ E. V. Sampathkumaran and A. Niazi, Phys. Rev. B 65, 180401(R) (2002); S. Rayaprol, K. Sengupta, and E. V. Sampathkumaran, ibid. 67, 180404(R) (2003).

${ }^{8}$ H. Kageyama, K. Yoshimura, K. Kosuge, H. Mitamura, and T. Goto, J. Phys. Soc. Jpn. 66, 1607 (1997).

${ }^{9}$ H. Kageyama, K. Yoshimura, K. Kosuge, M. Azuma, M. Takano,
H. Mitamura, and T. Goto, J. Phys. Soc. Jpn. 66, 3996 (1997).

${ }^{10}$ H. Kageyama, S. Kawasaki, K. Mibu. M. Takano, K. Yoshimura, and K. Kosuge, Phys. Rev. Lett. 79, 3258 (1997).

${ }^{11}$ A. Maignan, C. Michel, A. C. Masset, C. Martin, and B. Raveau, Eur. Phys. J. B 15, 657 (2000).

${ }^{12}$ B. Martínez, V. Laukhin, M. Hernando, J. Fontcuberta, M. Parras, and J. M. González-Calbet, Phys. Rev. B 64, 012417 (2001).

${ }^{13}$ B. Raquet, M. N. Baibich, J. M. Broto, H. Rakoto, S. Lambert, and A. Maignan, Phys. Rev. B 65, 104442 (2002).

${ }^{14}$ V. Hardy, S. Lambert, M. R. Lees, and D. McK. Paul, Phys. Rev. B 68, 014424 (2003).

${ }^{15}$ V. Hardy, M. R. Lees, A. Maignan, S. Hébert, D. Flahaut, C. Martin, and D. McK. Paul, J. Phys.: Condens. Matter 15, 5737 (2003).

${ }^{16}$ H. Fjellvag, E. Gulbrandsen, S. Aasland, A. Olsen, and B. Hauback, J. Solid State Chem. 124, 190 (1996).

${ }^{17}$ M. Mekata, J. Phys. Soc. Jpn. 42, 76 (1977); M. Mekata and K. Adachi, ibid. 44, 806 (1978).

${ }^{18}$ J. A. Osborn, Phys. Rev. 67, 351 (1945). 
${ }^{19}$ Note that, for the FD branch, the curve in $2.7 \mathrm{~T}$ (not shown for the sake of clarity) has the same shape as those recorded in $2 \mathrm{~T}$ or $2.3 \mathrm{~T}$ [see Fig. 5(a)].

${ }^{20}$ For a review, see Quantum Tunneling of Magnetization, edited by L. Gunther and B. Barbara (Kluwer, Dordrecht, 1995).

${ }^{21}$ J. R. Friedman, M. P. Sarachik, J. Tejada, and R. Ziolo, Phys. Rev. Lett. 76, 3830 (1996); L. Thomas, F. Lionti, R. Ballou, D. Gatteschi, R. Sessoli, and B. Barbara, Nature (London) 383, 145 (1996); E. M. Chudnovsky, Science 274, 938 (1996).
${ }^{22}$ A. Maignan, V. Hardy, S. Hébert, M. Drillon, M. R. Lees, O. Petrenko, D. McK. Paul, and D. Khomskii, J. Mater. Chem. 14, 1231 (2004).

${ }^{23}$ T. Takagi and M. Mekata, J. Phys. Soc. Jpn. 64, 4609 (1995).

${ }^{24}$ N. V. Prokof'ev and P. C. E. Stamp, Phys. Rev. Lett. 80, 5794 (1998); J. A. A. Perenboom, J. S. Brooks, S. Hill, T. Hathaway, and N. S. Dalal, Phys. Rev. B 58, 330 (1998); L. Thomas, A. Caneschi, and B. Barbara, Phys. Rev. Lett. 83, 2398 (1999). 